POS $\quad$ PROCEEDINGS

\title{
Current Status of the Namibian bid to host the Cherenkov Telescope Array
}

\author{
M. Backes* \\ University of Namibia, Department of Physics, Private Bag 13301, Windhoek, Namibia \\ E-mail: mbackes@unam.na
}

The Cherenkov Telescope Array (CTA) is the next generation instrument for very high energy (VHE) gamma-ray astronomy. Being successor to the vastly successful instruments H.E.S.S. in Namibia, MAGIC on the Canary Island of La Palma, and VERITAS in Arizona, USA, it is expected to outperform the former by a factor of 10, both in sensitivity as well as in the accessible energy range. To achieve these goals, the best possible operational conditions must be met and thus a world-wide site investigation campaign was launched. Based on the experience of successfully hosting the H.E.S.S. telescopes since 2002, proposals were submitted to host CTA in Namibia. Thorough investigations of the atmospheric and climatic conditions were carried out to estimate the average annual observation time. The scientific performance was estimated by means of Monte Carlo simulations, taking both the altitude and the local geomagnetic field into account. Eventually, the proposed site in Namibia was singled out as the scientifically best site in the world to host the CTA and in April 2014, the decision was taken to engage into official negotiations with the Republic of Namibia and with the European Southern Observatory (ESO), being patron to the competitor site in Chile. Details of the bidding process as well as the current status will be presented.

3rd Annual Conference on High Energy Astrophysics in Southern Africa-HEASA2015,

18-20 June 2015

University of Johannesburg, Auckland Park, South Africa

\footnotetext{
*Speaker.
} 


\section{Introduction}

The Cherenkov Telescope Array (CTA) [1] is the next-generation imaging atmospheric Cherenkov telescope (IACT) system that is in process of being designed for being built as of 2017. It will consist of two separate array, one in the Northern and one in the Southern hemisphere, respectively. IACTs operate in the very high energy (VHE; $>10 \mathrm{GeV}$ ) gamma-ray regime of the electromagnetic spectrum during clear and mostly moonless nights. The Northern hemisphere array is supposed to be comprised of 4 large-sized telescopes (LSTs) of 23 m-diameter [2] and 15 medium-sized telescopes (MSTs) of $12 \mathrm{~m}$-diameter [3], spread on an area of $1 \mathrm{~km}^{2}$. The baseline array for the Southern hemisphere spreads over $10 \mathrm{~km}^{2}$ and consists of 4 LSTs, 25 MSTs, and 70 small-sized telescopes (SSTs) of $6 \mathrm{~m}$-diameter [4]. Additionally, several medium-sized Schwarzschild-Couder (SC) telescopes of $9.5 \mathrm{~m}$-diameter [5] shall complement the Southern array. Figure 1 indicates the planed baseline layouts for the Northern and Southern site, respectively.
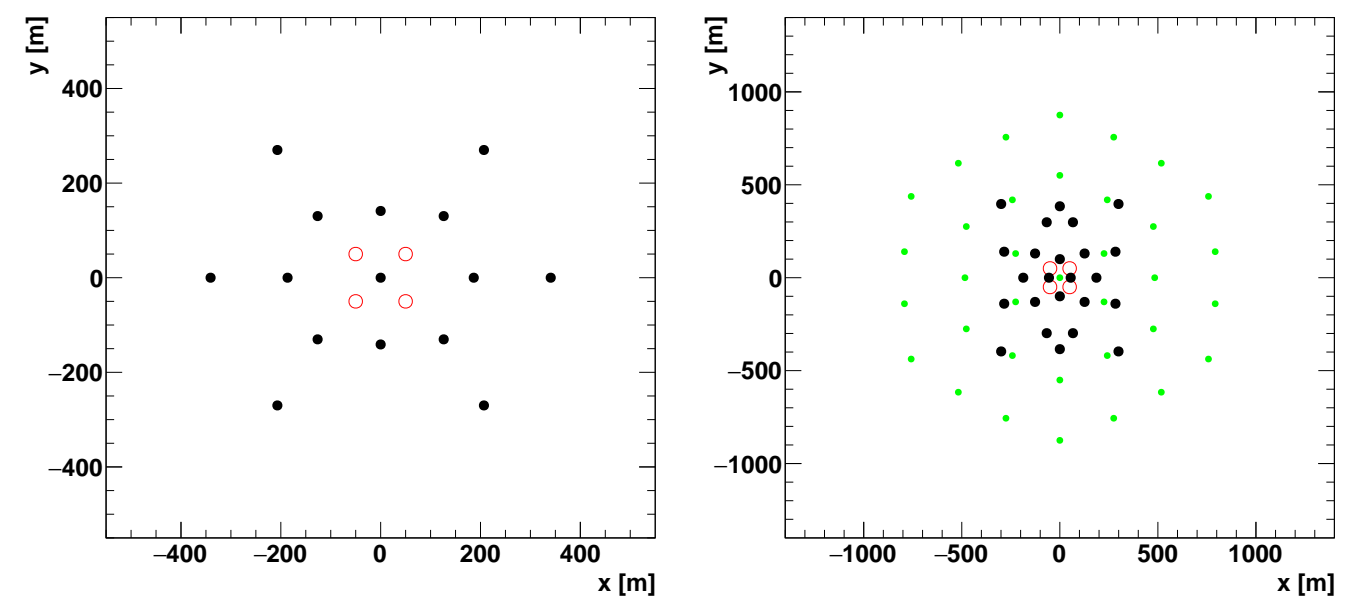

Figure 1: Reference layouts for the Northern (on the left) and Southern (on the right) CTA sites. Note the difference in scales of the axes between North and South. Red circles: large-sized telescopes; black markers: mid-sized telescopes; green markers: small-sized telescopes [6].

Particularly with the Southern array, CTA aims to outperform its predecessors H.E.S.S. [7], MAGIC [8], and VERITAS [9] by a factor of 10 in sensitivity and an increased energy coverage from a few tens of $\mathrm{GeV}$ to beyond $100 \mathrm{TeV}$ [10], cf. Fig. 2 .

The scientific contributions to construction and science operations of CTA will come from the CTA Consortium, which is formed by more than 1,200 scientists in 31 countries. A full list or countries can be obtained from [1] or [32].

\section{The CTA Site Search}

As CTA will be the world leading facility in VHE gamma-ray astronomy for an estimated lifetime of several decades, the decision on the where to place the two arrays is of utmost importance as the quality of a chosen site will affect the data quality and sensitivity of CTA during its entire lifetime. 


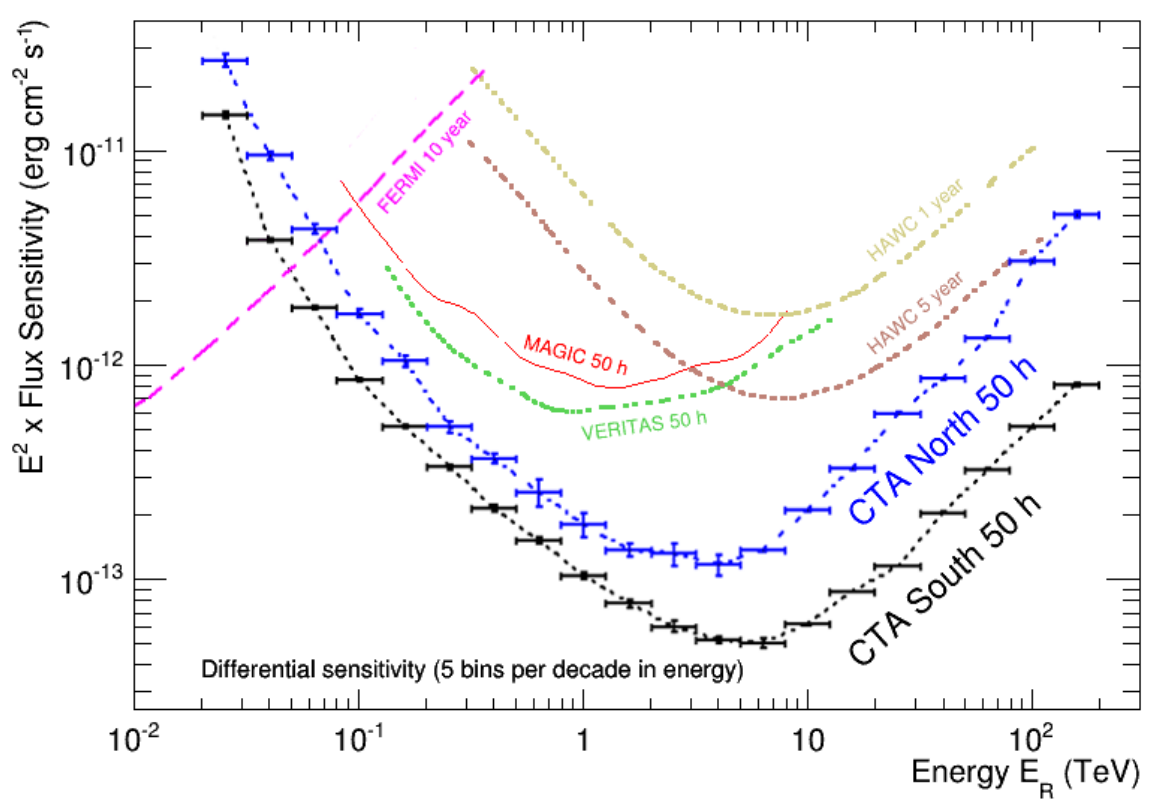

Figure 2: Differential sensitivity for a point-like $\gamma$-ray source of the Northern and Southern CTA arrays (for $50 \mathrm{~h}$ of observations) compared to current generation instruments [11].

\subsection{Initial Criteria and Call for Proposals}

In 2009, the initial requirements on the sites for the CTA were defined to be: 1) height $h$ above sea level, with $1,500 \mathrm{~m}$ a.s.l. $\leq h \leq 4,000 \mathrm{~m}$ a.s.l., 2) a flat area of $\left.3 \times 3 \mathrm{~km}^{2}, 3\right)>70 \%$ cloud free nights, 4) good atmospheric conditions in terms of high transparency, low humidity, modest wind speed, 5) light pollution $<1.5 \times$ the one at the current IACT sites, 6) latitude around $30^{\circ}$ North and South, respectively [12]. By these criteria, the regions highlighted in Fig. 3 were found.

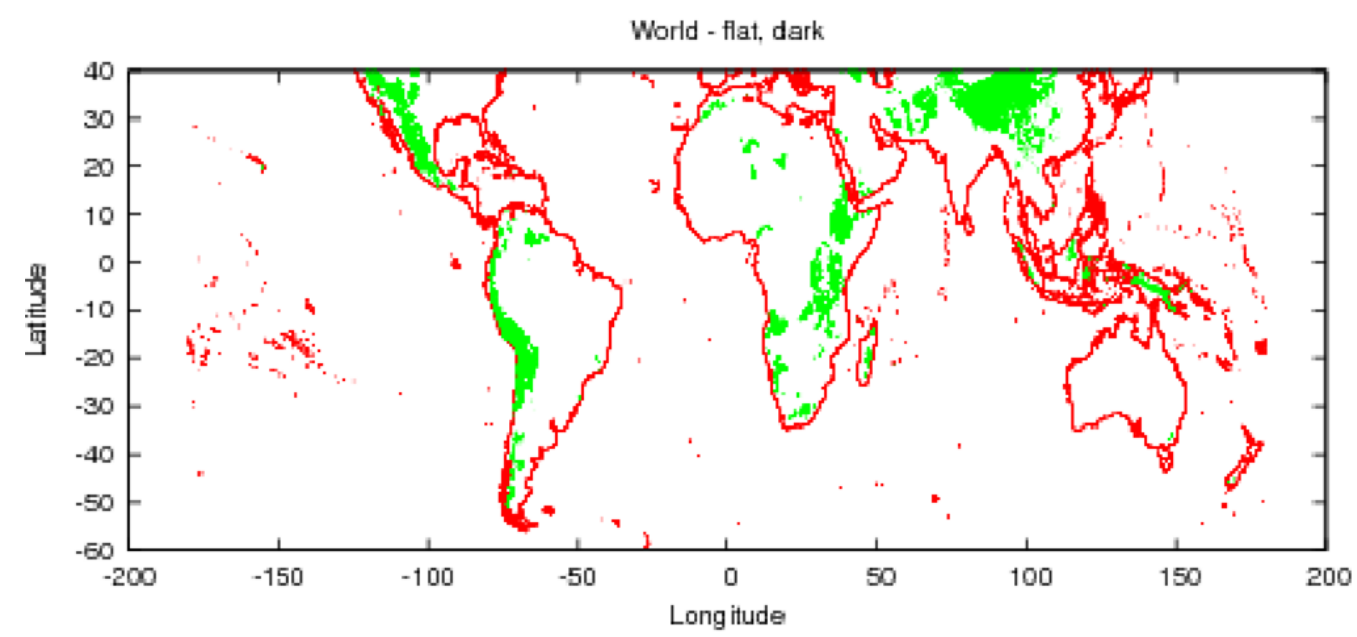

Figure 3: World map, showing (in green) areas fulfilling the criteria $h \geq 1,500 \mathrm{~m}$ a.s.1., maximal slope of $50 \mathrm{~m}$ on $1 \mathrm{~km}$, and low light pollution [12]. 
A refined analysis, limiting the maximum hight to 3,500 $\mathrm{m}$ a.s.1. and including information on the cloudiness narrowed down the possible regions to the ones highlighted in Fig. 4. Against this background, a call for proposals was issued, for CTA members to propose potential sites to host the CTA [13].

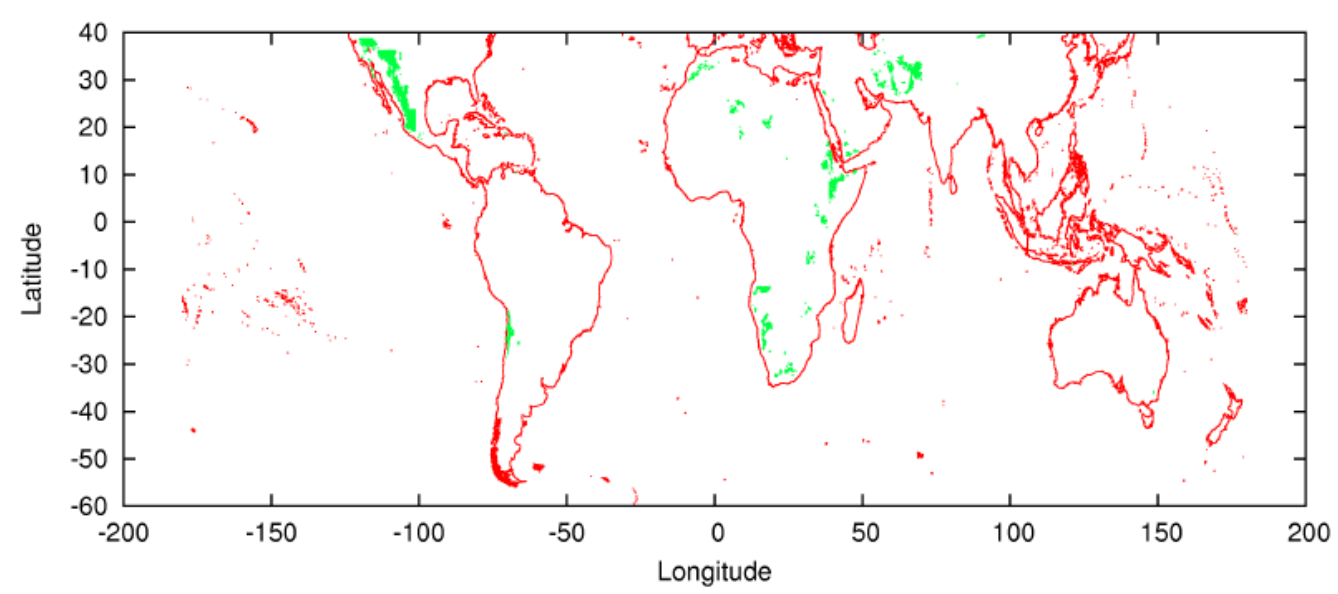

Figure 4: World map, showing (in green) areas fulfilling the criteria $1,500 \mathrm{~m}$ a.s.l. $\leq h \leq 3,500 \mathrm{~m}$ a.s.1., flat area of $10 \mathrm{~km}^{2}$, low light pollution, and sufficiently low cloudiness [13].

\section{Southern African Proposals and the site Aar in Namibia}

Following the call for proposals, two proposals were submitted from Southern Africa. The group at the University of Namibia proposed to host the CTA close to the well-known site of the H.E.S.S. telescopes in the Khomas Highland region, close to Mt. Gamsberg [14]. Emphasizing the long-known astronomical quality of the Gamsberg region, this proposal focused on the accessibility and infrastructure as well as the political support toward the project. At the same time, a group from the North-West University at Potchefstroom submitted a proposal, investigating in-depth cloudiness and weather conditions as well as geographical contours, and geological, political, and economical stability of several sites in Southern Africa [15]. Among the investigated sites were a site close to the South African Astronomical Observatory (SAAO) [16] in the Sutherland region in South Africa as well as sites in the Kuibis region, Karas, Namibia, including a site on the farm Aar. After two years of investigations, updated proposals were requested and a comprehensive proposal by the Namibian group, supported by South African scientists was submitted, focusing on both the Aar HESS and Aar sites, their respective cloudiness, weather, and geo-technical conditions, as well as accessing infrastructural conditions, construction and operation costs, natural as well as political risks, and local, national and regional political support [17].

Concerning socio-political risks in Namibia, one has to note that since independence in 1990, Namibia runs a stable democracy without any conflicts and with free elections ever since. This is also corroborated by the "B1" risk grade assigned by Euler-Hermes [18]. Crime rates, especially in rural areas of Namibia are very low, as indicated by the 2013 Numbeo crime and safety indexes for Namiba, which are comparable to those of the Netherlands [19]. 


\subsection{Site Aar}

As pointed out in [15], the potential sites in the Kuibis region, Karas, Namibia, lie in the least cloudy part of the subcontinent: with an average cloud cover of only $(13.3 \pm 0.7) \%$, and $(73.0 \pm 0.7) \%$ of clear night time, this region significantly outperforms the site of the South African Astronomical Observatory and even the current H.E.S.S. site, offering $(46.5 \pm 1.0) \%$ and $(64.4 \pm$ $1.4) \%$ of clear night time, respectively. The contour map of regional night-time cloud cover is depicted in Fig. 5.

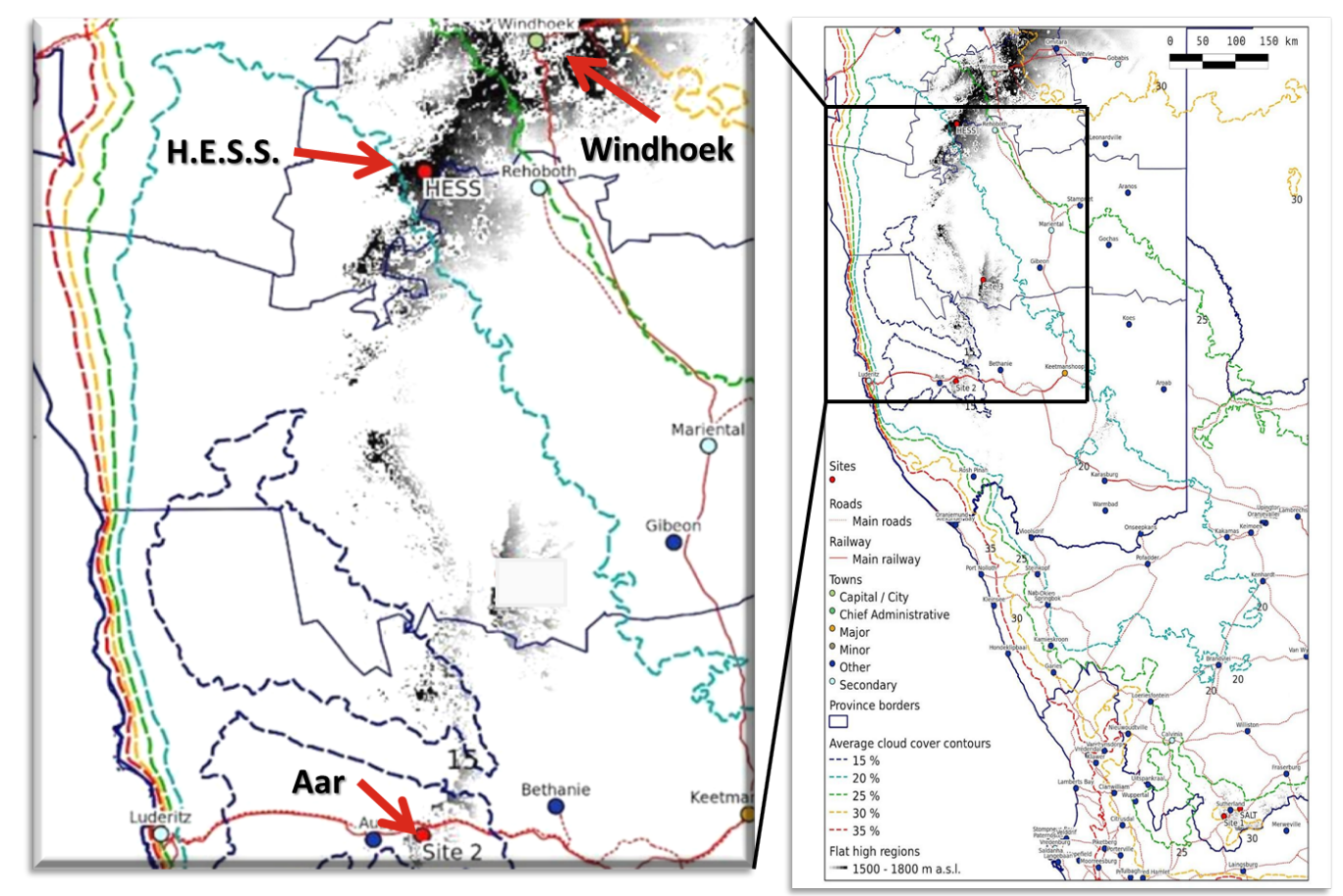

Figure 5: Southern African regional average night-time cloud cover contours. The gray-scale overlay indicates elevations of $\leq 1,500 \mathrm{~m}$ a.s.l. (white) $-\geq 1,800 \mathrm{~m}$ a.s.l. (black). The panel on the left, shows the region of central and Southern Namibia enlarged, including the H.E.S.S. and Aar sites [15].

In addition, Southern Namibia is one of the darkest regions of the sub-continent, as can be seen from Fig. 6.

Though the Kuibis region appears to be very remote, the region as well as the Aar site in particular, have very good accessibility, both for personnel and for cargo: The Lüderitz airport, about $120 \mathrm{~km}$ West of the Aar site offers a $1.8 \mathrm{~km}$ long tarred landing strip and four flights per week from and to Windhoek. By car, the Aar site can be reached within $7 \mathrm{~h}$ from Windhoek, driving the national tar roads B1 and B4. The close-by $(23 \mathrm{~km})$ town Aus offers a dirt airstrip cross of $1.4 \mathrm{~km} \times 1.1 \mathrm{~km}$ length that can be used by up to twin-engine small aircrafts. Furthermore, the Aus railway station offers a container handling facility, whereas the railway line is directly connected to the Lüderitz harbor, the second largest harbor in Namibia, capable of hosting ships up to $150 \mathrm{~m}$ length and handling containers up to $40 \mathrm{ft}$ and $36 \mathrm{t}$. 

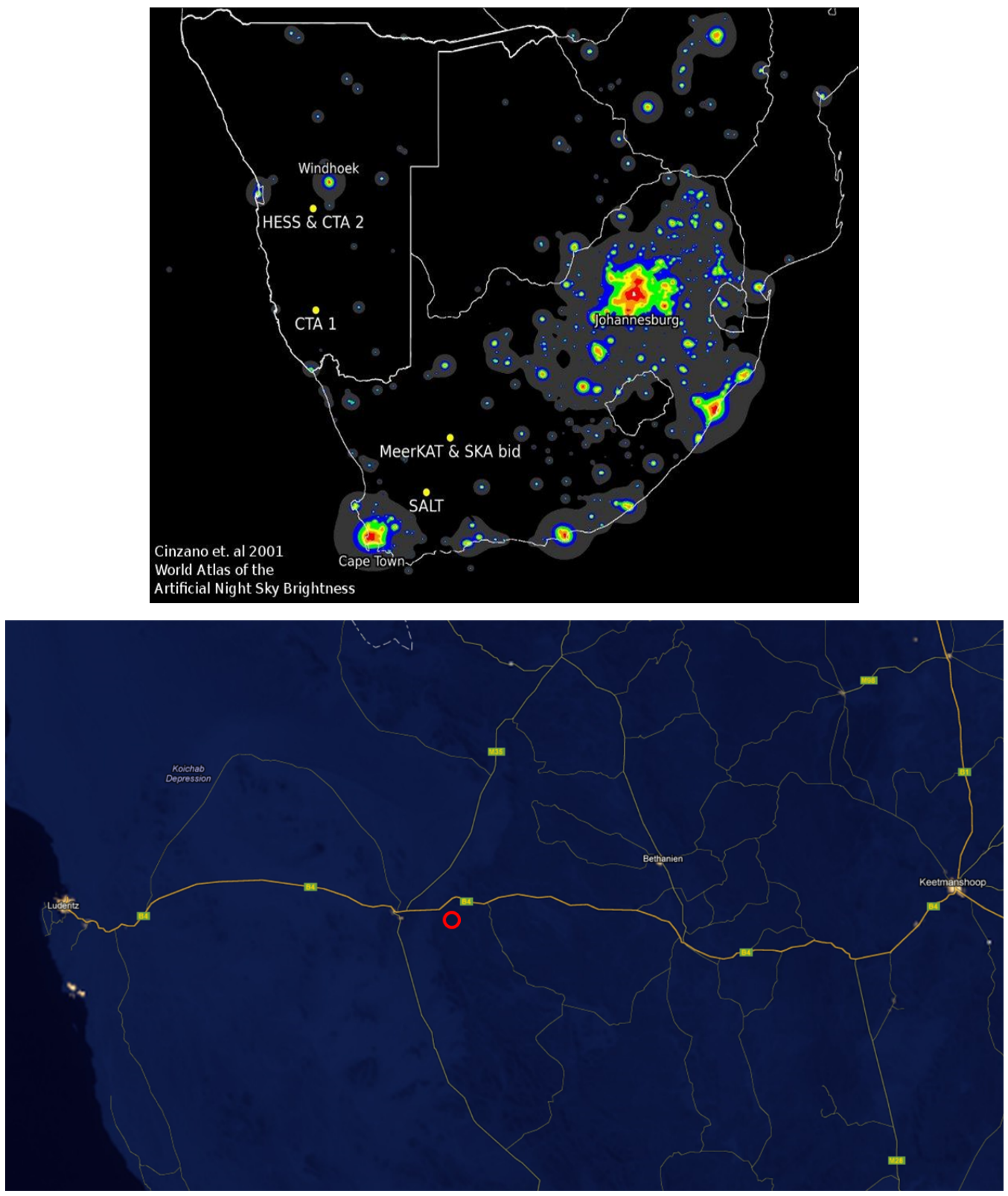

Figure 6: Top: Artificial night sky brightness for Southern Africa in astronomical V band, at zenith, for a clean atmosphere [20]. Bottom: Night-lights image of Southern Namibia. The coastal town Lüderitz as well as the regional capital Keetmanshoop can easily be identified. Lights from the town Aus at the road crossing central in the picture are bare seen and the Aar site, indicated by a red circle is completely dark [21].

In comparison to the other proposed sites in the Kuibis region [15], the Aar site was singled out as being close to all needed infrastructure, and thus must cost-effective: The site is only about $4 \mathrm{~km}$ away from the national tar road B4, and the closest substation of the national power grid $132 \mathrm{kV}$ line as well as of the national optical fiber backbone is only $20 \mathrm{~km}$ to West-North-West, from where a $33 \mathrm{kV}$ feeder and an optical fiber connection could easily be established. Furthermore, the railway town Aus is also only $23 \mathrm{~km}$ away. 


\section{Site Investigations and Ranking by the CTA Consortium}

In order to assess the submitted site proposals, the CTA Consortium investigated twofold the scientific quality of the proposed sites: On the one hand the average annual observation time (AAOT) was estimated and on the other hand, immense Monte Carlo simulations were conducted to estimate the performance per unit time (PPUT).

For estimating the AAOT, the atmospheric site characteristics were investigated: remote sensing data of the MODIS, METEOSAT, and GOES satellites were analyzed [22], atmospheric modeling was used to estimate long-term trends [23], where available, archival data measured by ground stations was analyzed and a dedicated instrument, the ATMOSCOPE, was developed and operated for more than a year at each site [24]. The ATMOSCOPE comprises a weather station, a cloud sensor a light of the night sky (LoNS) monitor a Unihedron Sky Quality Meter and an all-sky camera. The thorough analysis of all collected ATMOSCOPE data essentially supported the simulated cloud coverage of e.g. [23], testifying to the low cloudiness of the Namibian and Chilean sites, see the left panel of Fig. 9. Altogether, the site Aar in Namibia and the Armazones sites in Chile offer the highest AAOT of more than 1,200 $\mathrm{h}$ with low cloudiness conditions [25]. These numbers already account for losses due to high wind speeds and high aerosol optical density, see Fig. 7.

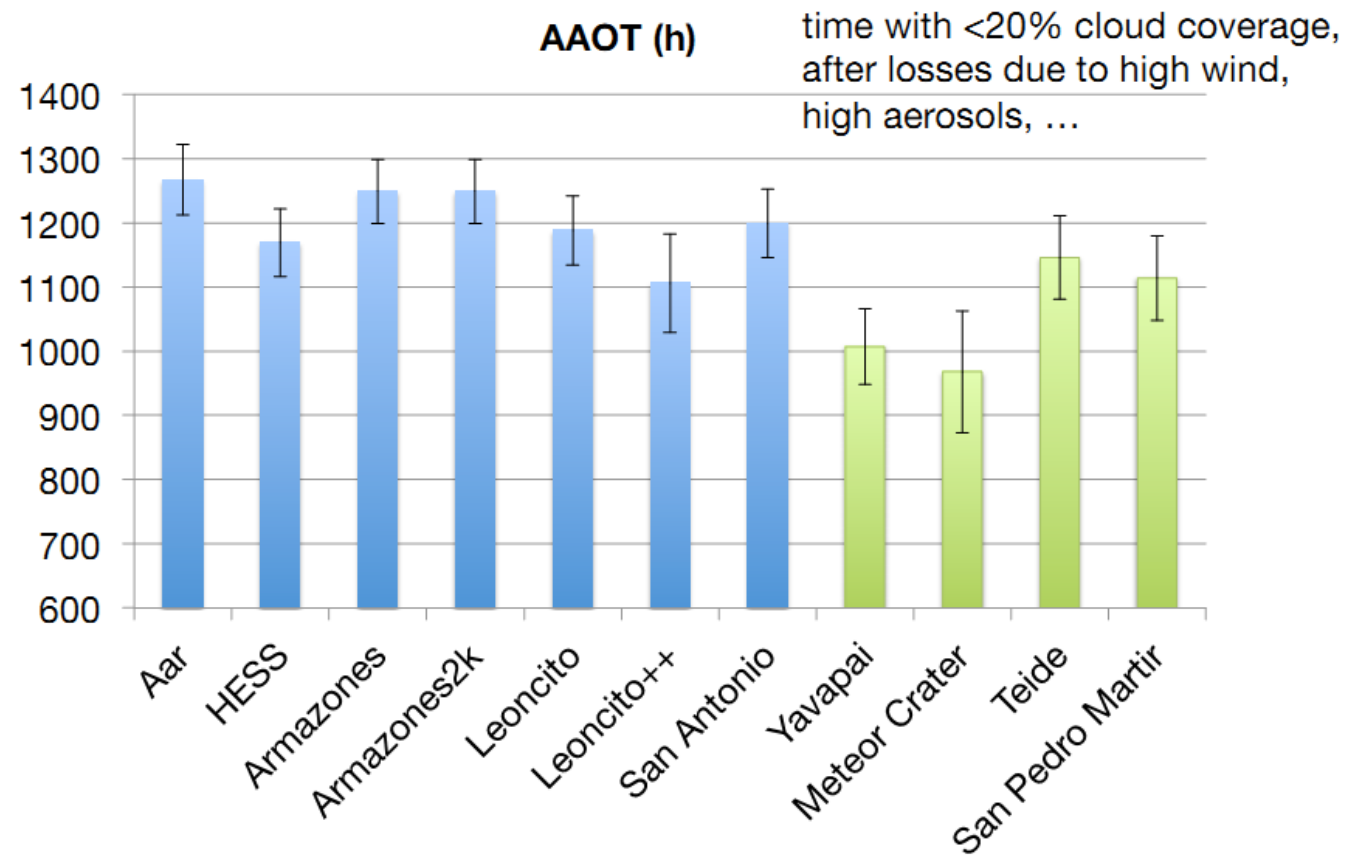

Figure 7: The average annual observation time (AAOT) with less than $20 \%$ cloud cover, corrected for losses due to high wind speed and high aerosol optical density. The Southern sites are indicated in blue, the Northern sites in green [25].

Monte Carlo simulations indicated that high altitude (3,700 $\mathrm{m}$ a.s.l.) sites are disfavored against lower altitude $(2,000 \mathrm{~m}$ a.s.l.) sites [26], except for the lowest energies $(<70 \mathrm{GeV})$, see the bottom left panel of Fig. 8. 


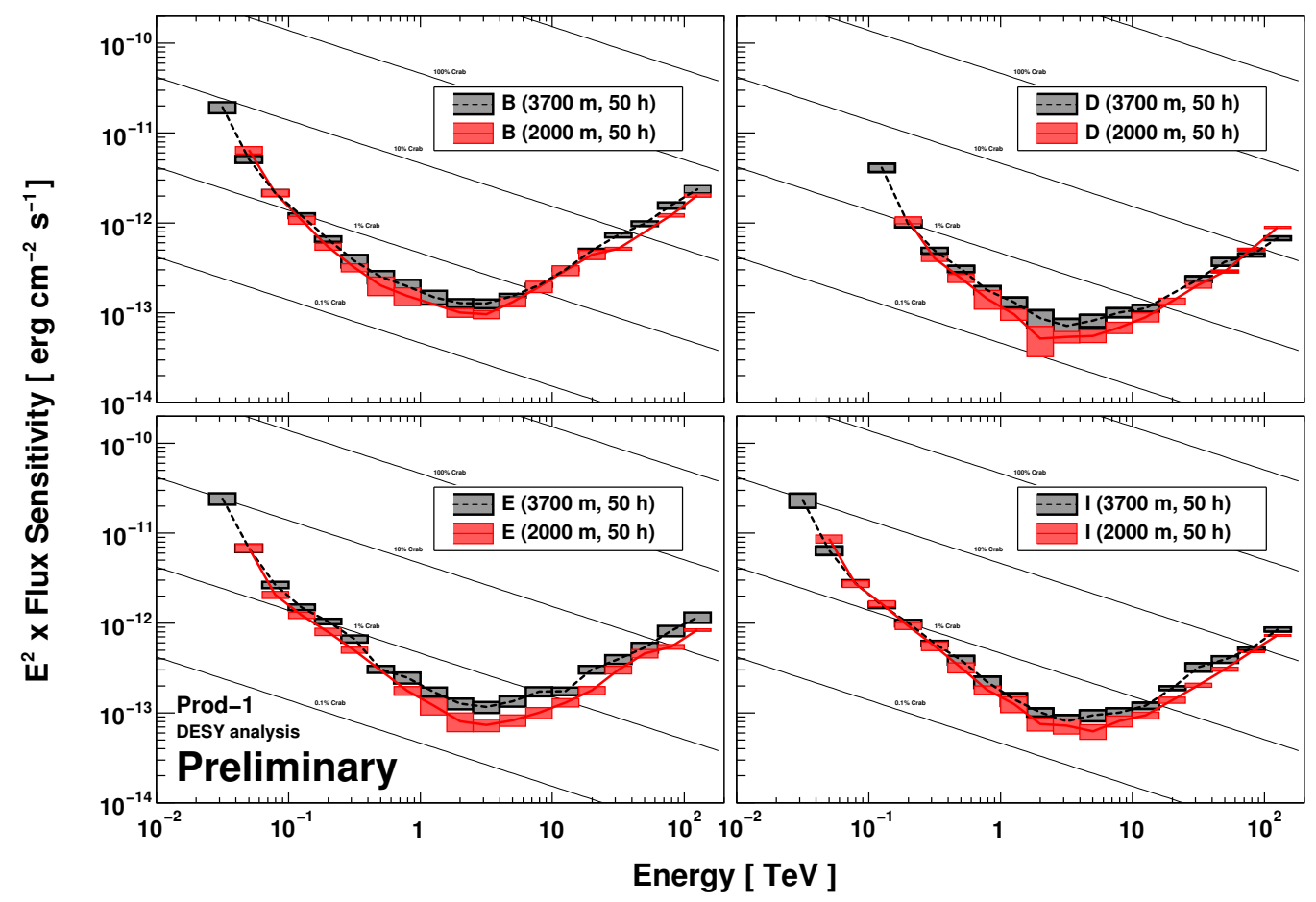

Figure 8: Differential sensitivity for different layout candidates for CTA at 2,000 $\mathrm{m}$ a.s.1. (red, solid line) and 3,700 $\mathrm{m}$ a.s.l. (black, dashed line) for $50 \mathrm{~h}$ of observations at $20^{\circ}$ zenith angle [26]. The lower left panel corresponds to the CTA South reference layout mentioned in the text.

Based on these findings, the CTA Consortium ranked the Aar site in Namibia the best site regarding scientific potential, whereas the Armazones site in Chile and the H.E.S.S. site in Namibia ranked second [27].

Additional Monte Carlo studies concerning the PPUT of the different sites, taking into account both, the different altitudes as well as the differences in the geo-magnetig field underlined the superior performance of the site Aar in Namibia [6]. The PPUT is calculated as the inverse geometric mean of the differential sensitivity, normalized by a reference sensitivity in each energy band:

$$
\text { PPUT }=\left(\prod_{N} \frac{F_{\text {sens, ref }}}{F_{\text {sens }}}\right)^{1 / N}
$$

with $N$ bins in energy (from $30 \mathrm{GeV}$ to $300 \mathrm{TeV}$ for CTA South, and to $20 \mathrm{TeV}$ for CTA North, respectively) of the reference flux sensitivity $F_{\text {sens, ref }}$ and the achieved flux sensitivity $F_{\text {sens. }}$. The right panel of Fig. 9 shows the altitude dependence of the PPUT for Northern and Southern pointing directions at the different sites, which clearly demonstrates the superior sensitivity at the site Aar compared to all other potential CTA sites in the Southern hemisphere.

Together with the high AAOT, these results single out site Aar as the scientifically best possible site to host the CTA. 

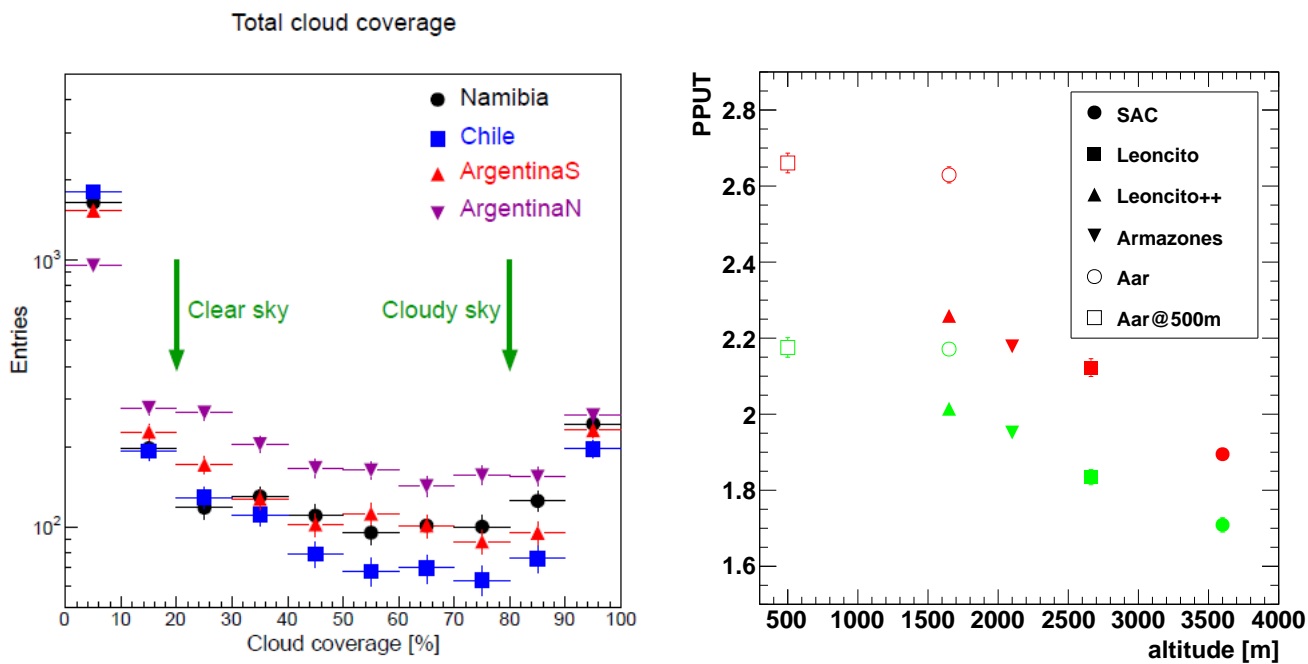

Figure 9: Left: Simulated cloud cover distributions for Southern hemisphere sites. An estimation of each value is provided every 3 hours [23]. Right: Performance per unit time (PPUT, see Eq. 4.1) as function of altitude for the Southern hemisphere candidate sites. Red-colored markers indicate results for the array pointing in Southern direction, green-colored markers for pointing in Northern direction [6].

\section{Decisions by the CTA Resource Board}

The governing body of CTA, the CTA Resource Board, decided on April 10, 2014 to start negotiations on the two sites in the Southern hemisphere, namely Aar in Namibia and European Southern Observatory (ESO) in Chile, keeping Argentina as possible back-up site [28], see also [29]. After a year of further investigations, the CTA Resource Board decided on March 25, 2015 to begin negotiations with two countries about the Northern hemisphere sites, namely Spain (La Palma) and Mexico (San Pedro Mártir), keeping the United States of America (Arizona) as back-up site [30], see Fig. 10.

\section{Recent Political Developments in Namibia}

Particularly in the context of CTA, SKA, and AVN, a joint bilateral ministerial meeting between Namibia and South Africa on Cooperation in Astronomy and Space Science was held on August 12, 2014 in Windhoek, Namibia. In the common statement [31], Hon. Mrs. G.M.N. Pandor, Minister of Science and Technology of the Republic of South Africa, and Hon. Dr. D. Namwandi, Minister of Education of the Republic of Namibia, communicate amongst others that

- both countries reaffirm their commitment towards strengthening cooperation in the fields of Astronomy and Space Science;

- South Africa reaffirms its political, scientific and technical support for Namibia's bid to host the CTA;

- both countries commit themselves to enhancing the human capacity for developing a globally competitive scientific and technical community in support of multi-wavelength Astronomy and Space Science in Southern Africa. 


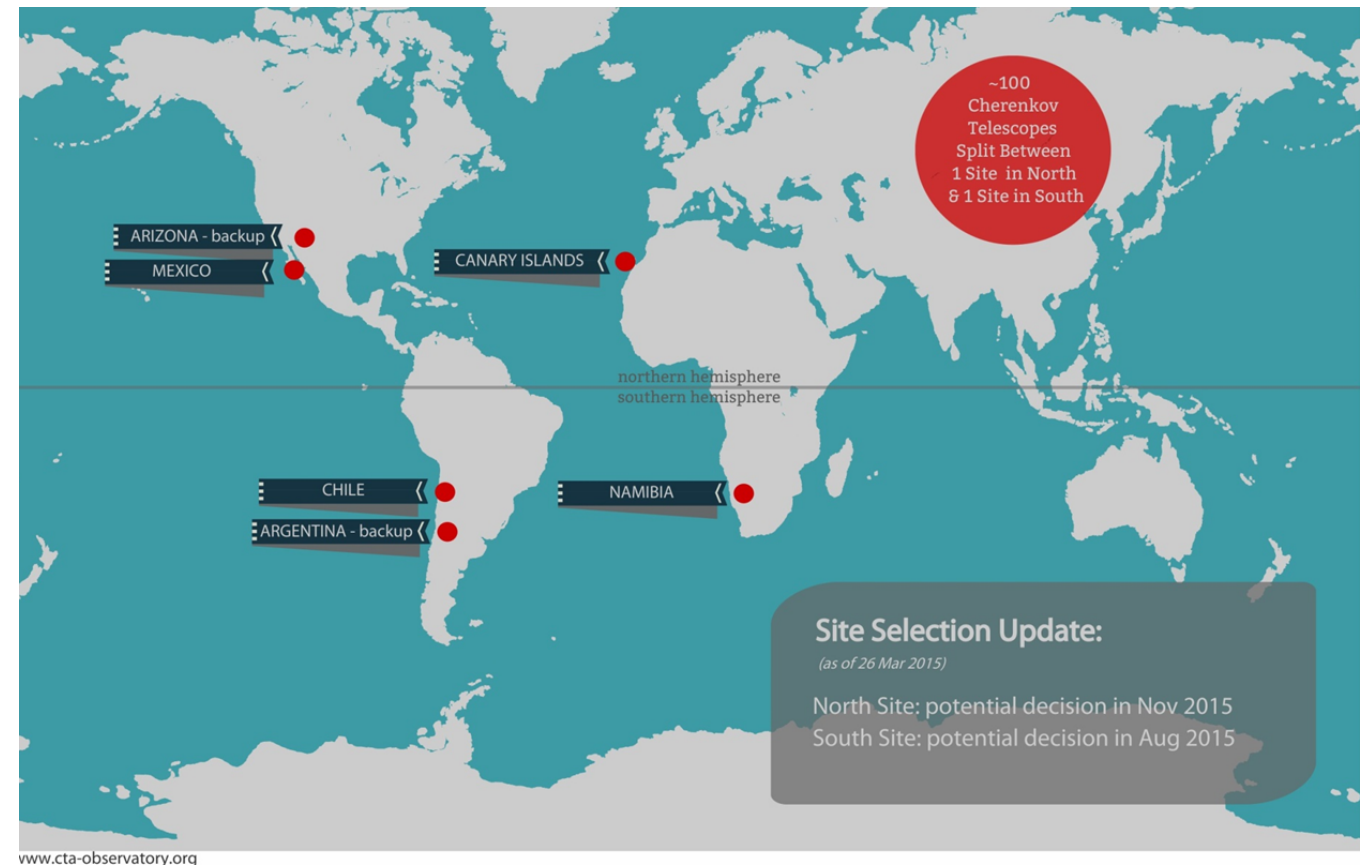

Figure 10: The sites selected by the CTA Resource Board as of March 2015 [30].

Meanwhile, the Cabinet of the Republic of Namibia officially approved support for Namibia to host the CTA. This includes sufficient funds for power, data transfer, water and road infrastructure to the edge of the proposed site within Namibia, as well as the purchase of the land for hosting the CTA by the National Commission on Research, Science and Technology (NCRST). Furthermore, in-kind contributions concerning custom duties, import levies, and VAT concessions were approved. It was also decided that the NCRST shall assume the leading role in coordinating CTA related activities in Namibia as well as leading the negotiations with the CTA Resource Board.

\section{Conclusion}

Altogether, the proposed Namibian site, Aar, shows world-leading scientific potential as Southern site of the CTA. This, together with the demonstrated national and regional political support makes it appear not unlikely that the CTA Resource Board will follow the recommendation by the CTA Consortium, and select Aar as Southern site for the CTA.

\section{Post-Conference Developments}

After the conclusion of this conference, on July 16, 2015, the CTA Resource Board took the decision to enter into detailed contract negotiations for hosting the CTA on the ESO Paranal grounds in Chile and at the Institutio de Astrofisica de Canarias (IAC), Roque de los Muchachos Observatory in La Palma, Spain. The Namibian and Mexican sites are kept as viable alternatives [32], see also [33]. 


\section{References}

[1] The Cherenkov Telescope Array - official website: http://www.cta-observatory.org

[2] J. Cortina et al., Status of the Cherenkov Telescope Array's Large Size Telescopes. In: Proceedings of Science PoS(ICRC2015)943: Proceedings of the 34th International Cosmic Ray Conference (ICRC2015), The Hague, The Netherlands. [arXiv:1508.06438].

[3] M. Garczarczyk et al., Status of the Medium-Sized Telescope for the Cherenkov Telescope Array. In: Proceedings of Science PoS(ICRC2015)959: Proceedings of the 34th International Cosmic Ray Conference (ICRC2015), The Hague, The Netherlands. [arXiv:1509.01361].

[4] T. Montaruli et al., The small size telescope projects for the Cherenkov Telescope Array. In: Proceedings of Science PoS(ICRC2015)1043: Proceedings of the 34th International Cosmic Ray Conference (ICRC2015), The Hague, The Netherlands. [arXiv:1508.06472].

[5] K. Byrum et al., A Medium Sized Schwarzschild-Couder Cherenkov Telescope Mechanical Design Proposed for the Cherenkov Telescope Array. In: Proceedings of Science PoS(ICRC2015)1029: Proceedings of the 34th International Cosmic Ray Conference (ICRC2015), The Hague, The Netherlands. [arXiv:1509.03074].

[6] G. Maier et al., Monte Carlo Performance Studies of Candidate Sites for the Cherenkov Telescope Array. In: Proceedings of Science PoS(ICRC2015)713: Proceedings of the 34th International Cosmic Ray Conference (ICRC2015), The Hague, The Netherlands. [arXiv:1508.06042].

[7] The High Energy Stereoscopic System - official website: http://www.mpi-hd.mpg.de/hfm/HESS

[8] The Major Atmospheric Gamma-ray Imaging Cherenkov Telescopes - official website: http://magic.mpp.mpg.de

[9] The Very Energetic Radiation Imaging Telescope Array System - official website: http://veritas.sao.arizona.edu

[10] B.S. Acharya et al., Introducing the CTA concept. In: Astroparticle Physics 43 (2013) 3-18. [DOI: 10.1016/j.astropartphys.2013.01.007].

[11] T. Hassan et al., Second large-scale Monte Carlo study for the Cherenkov Telescope Array. In: Proceedings of Science PoS(ICRC2015)971: Proceedings of the 34th International Cosmic Ray Conference (ICRC2015), The Hague, The Netherlands. [arXiv:1508.06075].

[12] M.C. Medina et al., Site Search for the Cherenkov Telescope Array (CTA) based on satellite data analysis. In: Proceedings of the 31st International Cosmic Ray Conference (ICRC2009), Łódź, Poland. http://icrc2009.uni.lodz.pl/proc/pdf/icrc1467.pdf

[13] T. Bulik et al., The CTA site search. In: Proceedings of the 32nd International Cosmic Ray Conference (ICRC2011), Beijing, China. [DOI: 10.7529/ICRC2011/V09/1257].

[14] R. Steenkamp and I.D. Davids, Namibian Site Proposal. In: CTA internal note (2011).

[15] P.P. Krüger and D.J. van der Walt, Southern Africa CTA Site Proposal. In: CTA internal note (2011). [arXiv:1202.4747].

[16] The South African Astronomical Observatory - official website: http://www.saao.ac.za

[17] M. Backes et al., Candidate Sites for the Cherenkov Telescope Array in Namibia. In: CTA internal note (2013). 
[18] A. Atkinson and Euler Hermes Economic Research Team, Country Report Namibia (2014), http://www.eulerhermes.com/mediacenter/Lists/mediacenter-documents/Country-Report-Namibia.pdf

[19] Numbeo, Crime Index for Country 2013, http://www.numbeo.com/crime/rankings_by_country.jsp?title=2013-Q1

[20] P. Cinzano et al., The first world atlas of the artificial night sky brightness. In: Monthly Notices of the Royal Astronomical Society 328 (2001) 689. [DOI: 10.1046/j.1365-8711.2001.04882.x]. [arXiv:astro-ph/0108052].

[21] U. Enke, Blue Marble Night-lights imaginary by NASA's Earth Observatory (2012), http://www.blue-marble.de/nightlights/2012. In: Science 311 (2006) 1529: http://www.sciencemag.org/content/311/5767/1529.5.full.pdf

[22] S. Vincent et al., Atmospheric considerations for the CTA site search. In: Proceedings of the Armospheric Monitoring for High-Energy Astroparticle Detectors (AtmoHEAD) Conference 2013, Saclay, France. [arXiv:1403.5075].

[23] K. Louedec and M. Will, Atmospheric Considerations for CTA site search using global models. In: Journal of Physics: Conference Series, 409/1 (2013) id. 012121. [DOI: 10.1088/1742-6596/409/1/012121], [arXiv:1207.3534].

[24] C. Fruck et al., Instrumentation for comparing night sky quality and atmospheric conditions of CTA site candidates. In: Journal of Instrumentation, 10/04 (2015) P04012. [DOI: 10.1088/1748-0221/10/04/P04012], [arXiv:1501.02156].

[25] W. Hofmann, Status of CTA. In: Presentation at the 5th Workshop on Air Shower Detection at High Altitude, 26-28 May 2014, Paris, France. http://highaltitude.sciencesconf.org/conference/highaltitude/pages/CTA_Paris_2014_05_Hofmann.pdf

[26] K. Bernlöhr et al., Progress in Monte Carlo design and optimization of the Cherenkov Telescope Array. In: Proceedings of the 33rd International Cosmic Ray Conference (ICRC2013), Rio de Janeiro, Brazil. http://www.cbpf.br/ icrc2013/papers/icrc2013-1053.pdf [arXiv:1307.2773].

[27] N. Dominguez, Namibia Gets Top Grade as Base for Major Gamma Ray Telescope. In: ScienceInsider. http://news.sciencemag.org/physics/2013/10/namibia-gets-top-grade-base-majorgamma-ray-telescope

[28] The Cherenkov Telescope Array, Gamma-Ray Astronomy: Site Negotiations for Cherenkov Telescope Array Started. In: official news release: https://portal.cta-observatory.org/SiteAssets/Pages/News/SiteNegotiationsStarted_10042014.pdf

[29] E. Gibney, Panel homes in on sites for $\gamma$-ray detector. In: Nature 508 (2014) 297. [DOI: 10.1038/508297a].

[30] The Cherenkov Telescope Array, Start of Negotiations for CTA North. In: official news release: https://portal.ctaobservatory.org/SiteAssets/Pages/News/NorthSitesNarrowed_26032015_updated.pdf

[31] Hon. G.M.N. Pandor and Hon. D. Namwandi, Statement of the Joint Bilateral Ministerial Meeting between Namibia and South Africa on Cooperation in Astronomy and Space Science. 2014

[32] The Cherenkov Telescope Array, Paranal and La Palma Sites Chosen for Final Negotiations to Host World's Largest Array of Gamma-Ray Telescopes. In: official news release: https://portal.ctaobservatory.org/SiteAssets/Pages/News/Paranal\%20and\%20La\%20Palma\%20Sites_07162015_v2.pdf

[33] C. Cesare, Spain and Chile chosen to host $\gamma$-ray telescope. In: Nature (2015). [DOI: 10.1038/nature.2015.18008]. 